\title{
Non-Diabetic Renal Disease in Patients with Type 2 Diabetes Mellitus with Proteinuria
}

\author{
Manik Chandra Mondal1 ${ }^{*}$, Rana Mokarrom Hossain'1, K. B. M. Hadiuzzaman', Md. Omar Faroque ${ }^{1}$, \\ Shah Md. Zakir Hossain'1, A. K. M. Shahidur Rahman'1, Fahad Al Shatil Ashrafee1, Popy Sarker², \\ Abu Zafor Md. Salahuddin ${ }^{3}$, Mohammad Kamrul Ahsan ${ }^{4}$

\footnotetext{
${ }^{1}$ Department of Nephrology, Bangabandhu Sheikh Mujib Medical University (BSMMU), Dhaka, Bangladesh

${ }^{2}$ Department of Orthodontics and Dento-Facial Orthopaedics, Dhaka Dental College and Hospital (DDCH), Dhaka, Bangladesh

${ }^{3}$ Department of Nephrology, Mymensingh Medical College Hospital (MMCH), Mymensingh, Bangladesh

${ }^{4}$ Holy Family Red Crescent Medical College Hospital (HFRCMCH), Dhaka, Bangladesh

Email: *manikrmc40@yahoo.com
}

How to cite this paper: Mondal, M.C., Hossain, R.M., Hadiuzzaman, K.B.M., Faroque, Md.O., Hossain, S.Md.Z., Shahidur Rahman, A.K.M., Ashrafee, F. Al S., Sarker, P., Salahuddin, A.Z.Md. and Ahsan, M.K. (2019) Non-Diabetic Renal Disease in Patients with Type 2 Diabetes Mellitus with Proteinuria. Journal of Biosciences and Medicines, 7, 68-78.

https://doi.org/10.4236/jbm.2019.712007

Received: November 1, 2019

Accepted: December 1, 2019

Published: December 4, 2019

Copyright (c) 2019 by author(s) and Scientific Research Publishing Inc. This work is licensed under the Creative Commons Attribution International License (CC BY 4.0).

http://creativecommons.org/licenses/by/4.0/

\section{Open Access}

\begin{abstract}
Background: Diabetes mellitus (DM) is the leading cause of end stage renal disease (ESRD) worldwide. Although DM with proteinuria is the ultimate result of diabetic nephropathy (DN), a wide spectrum of non-diabetic renal diseases (NDRD) can occur in such patients. Objective: To observe the frequency and histological pattern of NDRD in diabetic patients with proteinuria and to explore their association with clinical and laboratory parameters. Methods: This cross-sectional study was conducted in the Department of Nephrology, Bangabandhu Sheikh Mujib Medical University (BSMMU), Dhaka, Bangladesh from April 2016 to September 2017. In this study a total of 38 cases of DM with proteinuria ( $>1$ gm/24-hour) were selected purposively. Renal biopsy was done in all patients. Based on histological findings they were categorized into two groups; Group 1 with NDRD and Group II with DN. Their clinical and laboratory parameters were analyzed and compared. Results: Among the total study subjects, 21 (55.3\%) were male and 17 $(44.7 \%)$ were female, mean $( \pm S D)$ age $43.45 \pm 9.99$ years in the NDRD group and $41.57 \pm 9.50$ years in the DN group. Thirty one cases $(81.6 \%)$ out of thirty eight had NDRD and seven (18.4\%) cases had isolated DN; therefore more than two third cases had NDRD. Duration of DM was found to be significantly shorter $(\mathrm{p}=0.004)$ in the NDRD group. Diabetic retinopathy was present in $12.9 \%$ cases in NDRD group vs. $57.1 \%$ cases in DN group ( $\mathrm{p}=$ 0.025). Frequency of microscopic hematuria was significantly higher (90.3\%) in NDRD patients $(\mathrm{p}=0.002)$. Conclusion: The frequency of NDRD in type 2 diabetic patients other than diabetic nephropathy is relatively high. Membrano proliferative glomerulonephritis and membranous nephropathy are
\end{abstract}


more common in NDRD. Absence of diabetic retinopathy, presence of hematuria and shorter duration of DM are markers associated with NDRD in type $2 \mathrm{DM}$, which are important indicators for renal biopsy in diabetic patients with proteinuria.

\section{Keywords}

Diabetes Mellitus (DM), Diabetic Nephropathy (DN), Non-Diabetic Renal Disease (NDRD), Renal Biopsy

\section{Introduction}

Diabetes mellitus (DM) is a silent killer affecting several organs with a wide range of microvascular and macrovascular complications. Worldwide both incidence and prevalence of diabetes mellitus are increasing and its most alarming complication diabetic nephropathy (DN) raising rapidly especially in developing countries [1] [2] [3].

In the case of diabetes mellitus, diabetic nephropathy is almost always diagnosed clinically based on a long history of diabetes, presence of other microvascular complications and evidenced by proteinuria prior to renal failure [4]. However apart from diabetic nephropathy there are other diseases that affect the kidneys in diabetic patients known as non diabetic renal disease (NDRD) that can be confirmed by renal biopsy [4].

It has been histologically proven that $>95 \%$ of patients with renal disease have type-1 DM of at least 5 years duration along with diabetic neuropathy and retinopathy, and the primary renal disease is in fact diabetic nephropathy. However in type 2 DM, nephropathy may be found at the initial diagnosis of DM [5]. Diabetic retinopathy is very common and hence sensitive in case of diabetic nephropathy with type $1 \mathrm{DM}$ but not as sensitive or specific for diabetic nephropathy in type $2 \mathrm{DM}[6]$.

Around $12 \%$ - $81 \%$ of type-2 DM cases have been seen to have NDRD either isolated or superimposed on diabetic nephropathy on renal biopsy specimens [7]. Renal biopsy is thus crucial for making such specific diagnosis, planning of management and assessing prognosis [8]. Renal biopsy specimens require histologic examination as well as immunofluorescence study. Few studies reported that NDRD ranges from $27 \%$ to $79 \%$ including both glomerular and tubule-interstitial lesions [9].

Progression of diabetic nephropathy (DN) can be slowed, but certain non diabetic renal disease (NDRD) such as mesangial proliferative glomerulonephritis, membranous nephropathy etc. can often remit on treatment [10]. As the treatment and prognosis of these two entities are different, so it is very important for correct diagnosis and differentiating between these two in diabetic subjects.

Features consistent with the natural history of NDRD include a short duration of DM ( $<5$ years), absence of diabetic retinopathy, early appearance of overt 
proteinuria, rapid decline in renal function or impaired renal function without significant proteinuria. The predictive values of these criteria are quite variable, so renal biopsy is required to confirm the diagnosis [11].

The occurrence of isolated NDRD or with concurrent DN has important implications on therapy and prognosis, because NDRD is often treatable. Early diagnosis of NDRD helps in starting appropriate therapy which could aid in prolonging renal survival among this population.

Patients with NDRD or NDRD superimposed on DN respond better to specific therapies rather than angiotensin converting enzyme inhibitor or angiotensin receptor blocker alone, that are used in DN. This emphasizes to suspect, investigate and treat proteinuric diabetic patients with an atypical clinical course as early as possible [12] [13].

Therefore, this study was conducted to observe the frequency of NDRD in diabetic patients having proteinuria, along with histological pattern and their association with clinical and laboratory parameters.

\section{Methods}

This cross-sectional study was carried out in the Department of Nephrology, Bangabandhu Sheikh Mujib Medical University (BSMMU), Dhaka, Bangladesh from April 2016 to September 2017. The study was approved by the Ethical Review Committee, BSMMU, Dhaka, Bangladesh. According to the statistical calculation final sample was 54 . A total of 54 diabetic patients were initially taken in suspicion of NDRD but 16 patients refused to do renal biopsy leaving 38 patients that remained to be analyzed in this study. Therefore a total of thirty eight (38) patients were selected purposively. Informed written consent was taken from each participant prior to enrollment. Diabetic patients who gave history suggestive of non-diabetic renal disease were evaluated by physical examination and laboratory investigations. In all patients; urine routine microscopic examination (R/M/E), 24-hour urinary total protein (UTP), phase contrast microscopy of urine (if red cells were present in urine), hemoglobin level, serum creatinine, serum albumin, fasting blood glucose (FBS) and 2-hour postprandial blood sugar (2HABF) along with glycosylated hemoglobin (HbAlc), HBsAg, Anti-HCV, complement levels $(\mathrm{C} 3, \mathrm{C} 4)$ and ultrasonography of KUB (kidney, ureter and bladder) region were done. In selected cases ANA, Anti-dsDNA antibody and Anti-Neutrophil Cytoplasmic Antibodies were also done. Urine for culture and sensitivity was done to exclude any urinary tract infections. X-ray KUB region was done to exclude renal stone disease. Consultation was taken from an ophthalmologist, Department of Ophthalmology, BSMMU for evidence of diabetic retinopathy. The participants were divided into two groups based on the histological evidence: NDRD group and DN group. The outcome variables analysed and compared between the two groups; these were age (yrs), gender (M/F), duration of diabetes mellitus (yrs), FBS (mmol/L), $2 \mathrm{HABF}(\mathrm{mmol} / \mathrm{L})$ and $\mathrm{HbAlc}$ (\%), haematuria. serum creatinine ( $\mathrm{mg} / \mathrm{dl})$, hemoglobin level $(\mathrm{gm} / \mathrm{dl})$, serum al- 
bumin $(\mathrm{mg} / \mathrm{dl})$, 24-hours urinary total protein (gm/24-hour) and diabetic retinopathy.

Diabetic patients with proteinuria (>1 gm/24-hour) along with any of the following criteria: absence of diabetic retinopathy, rapidly declining renal function, recent onset of nephrotic range proteinuria, unexplained hematuria or any other active urinary sediments underwent percutaneous renal biopsy after all pre-biopsy work up. Two biopsy samples were sent to the Department of Pathology, BSMMU in two separate test tubes; one test tube containing biopsy material in formalin for light microscopy and another test tube containing biopsy material in normal saline for immunofluorescence study. Biopsy samples were analyzed by a single renal histopathologist using Hematoxylin and Eosin stain, Periodic Acid Schiff stain (PAS) and Silver stain for light microscopy. Immunofluorescence study was also done. After obtaining the histopathology reports the study subjects were analyzed in two groups: non-diabetic renal disease (NDRD) and diabetic nephropathy (DN). Patients with mixed lesions were categorized as non-diabetic renal disease.

Statistical analysis was performed using SPSS (Statistical Package for Social Science) version 22. Statistical differences between the NDRD and DN groups were calculated by unpaired t-test for quantitative variables and Fisher Exact test for qualitative variables. Multiple regression analysis was done where appropriate. We adopted $5 \%$ as significant level.

\section{Results}

A total of thirty eight (38) patients were selected purposively to be analyzed in this study. Among them 21 (55.3\%) patients were male and 17 (44.7\%) patients were female.

Thirty one cases (81.6\%) out of thirty eight had NDRD (placed into Group-I) and seven (18.4\%) cases had isolated DN (placed into Group-II), therefore more than two third cases had NDRD in this study (Table 1).

Mean age of the patients were $43.45 \pm 9.99$ years in NDRD group and $41.57 \pm$ 9.50 years in DN group, ratio between male and female was 1.0:0.93 and 1.0:0.40 respectively. The observed differences were not statistically significant (Table 2).

Table 3 shows total NDRD and DN subjects. NDRD was found more frequent $(81.6 \%)$ in the study population. Among NDRD group; membranoproliferative glomerulonephritis was most common 8 (21.1\%) cases, followed by membranous nephropathy 7 (18.4\%), diffuse proliferative glomerulonephritis 6 (15.8\%), mesangial proliferative glomerulonephritis 5 (13.2\%), focal segmental glomerulosclerosis 3 (7.9\%), IgA nephropathy 1 (2.6\%) and minimal change disease (MCD) 1 (2.6\%) cases.

Table 4 shows that a shorter duration ( $<5$ years) of DM was found in 29 cases, among which 27 (87.1\%) cases had NDRD and 2 (28.6\%) cases had DN. Duration of DM was longer ( $>5$ years) in most cases $(71.4 \%)$ of the diabetic nephropathy $(\mathrm{DN})$ group. There was significant difference $(\mathrm{p}=0.004)$ in duration of DM between NDRD and DN groups. 
Table 1. Pattern of renal disease in the study subjects $(n=38)$.

\begin{tabular}{ccc}
\hline Lesions & Frequency (n) & Percentage (\%) \\
\hline Group I: NDRD & 31 & 81.6 \\
Group II: Isolated DN & 7 & 18.4 \\
Total & 38 & 100.0 \\
\hline
\end{tabular}

NDRD—Non diabetic renal disease, DN—Diabetic nephropathy.

Table 2. Demographic status among different types of renal disease $(n=38)$.

\begin{tabular}{cccc}
\hline \multirow{2}{*}{ Variables } & \multicolumn{2}{c}{ Groups } & p value \\
\cline { 2 - 3 } & NDRD & DN & \\
\hline Age (years) & $43.45 \pm 9.99$ & $41.57 \pm 9.50$ & $0.653^{*}$ \\
Gender (M:F) & $1.0: 0.93$ & $1.0: 0.40$ & $0.427^{*}$ \\
\hline
\end{tabular}

NDRD—Non diabetic renal disease, DN—Diabetic nephropathy; ${ }^{\star}$ Unpaired "t" test and Fisher Exact test was done to measure the level of significance.

Table 3. Distribution of patients according to type of renal histological pattern $(n=38)$.

\begin{tabular}{ccc}
\hline & Frequency $(\mathrm{n})$ & Percentage $(\%)$ \\
\hline NDRD & 31 & $\mathbf{8 1 . 6}$ \\
Membrano-proliferative GN & 8 & 21.1 \\
Membranous nephropathy & 7 & 18.4 \\
Diffuse proliferative GN & 6 & 15.8 \\
Mesangial proliferative GN & 5 & 13.2 \\
Focal segmental glomerulosclerosis & 3 & 7.9 \\
IgA nephropathy & 1 & 2.6 \\
Minimal change disease (MCD) & 1 & 2.6 \\
DN & 7 & 18.4 \\
\hline
\end{tabular}

NDRD—Non diabetic renal disease, DN—Diabetic nephropathy, GN-Glomerulonephritis.

Table 4. Distribution of patients in relation to duration of diabetes mellitus $(n=38)$.

\begin{tabular}{cccc}
\hline \multirow{2}{*}{ Duration of DM (years) } & \multicolumn{2}{c}{ Groups } & \multirow{2}{*}{ p value } \\
\cline { 2 - 3 } & NDRD & DN & $0.004^{*}$ \\
\hline 5 & $27(87.1 \%)$ & $2(28.6 \%)$ & \\
Total & $4(12.9 \%)$ & $5(71.4 \%)$ & \\
\hline
\end{tabular}

DM-Diabetes mellitus, NDRD-Non diabetic renal disease, DN-Diabetic nephropathy. Values in the parenthesis denote corresponding percentage. ${ }^{*}$ We used Fisher's exact test as statistical method.

Table 5 shows that serum creatinine, hemoglobin level and serum albumin was relatively high in diabetic nephropathy (DN) group. Urinary total protein was relatively high in non diabetic renal disease (NDRD) group. These observed differences were not approaching to the level of significance. 
Table 6 shows the glycemic status of study subjects. Mean $( \pm S D)$ fasting blood sugar (FBS-mmol/L), 2-hour after postprandial blood sugar (2HABF-mmol/L) and glycosylated hemoglobin (HbAlc-\%) among NDRD patients was $7.69 \pm 3.07$ $\mathrm{mmol} / \mathrm{L}, 11.20 \pm 3.27 \mathrm{mmol} / \mathrm{L}$ and $7.35 \pm 1.42 \%$ which was $8.23 \pm 3.68 \mathrm{mmol} / \mathrm{L}$, $9.95 \pm 2.40 \mathrm{mmol} / \mathrm{L}$ and $7.65 \% \pm 1.03 \%$ among DN patients respectively. Therefore it has been observed that there was no significant difference in glycemic status between NDRD and DN groups.

Out of total 38 cases; diabetic retinopathy was absent in 30 cases, of whom 27 (90\%) cases had NDRD and $3(10 \%)$ cases had DN. Absence of diabetic retinopathy therefore turned out to become a significant predictor of non-diabetic renal disease $(\mathrm{p}=0.025)$ (Table 7$)$.

In this study hematuria was present in 30 cases among total 38 study subjects, of them 28 cases had NDRD and only 2 cases had DN as shown in Table 8. Thus it has been observed that hematuria was significantly frequent in the NDRD group ( $\mathrm{p}=0.002)$.

Table 9 shows that, absence of diabetic retinopathy was the most significant predictor of non-diabetic renal disease among diabetic patients presenting with proteinuria $(\mathrm{p}=0.026)$.

Table 5. Laboratory findings of study subjects.

\begin{tabular}{cccc}
\hline \multirow{2}{*}{ Variables } & \multicolumn{2}{c}{ Groups } & \multirow{2}{*}{ p value } \\
\cline { 2 - 3 } & NDRD & DN & $0.097^{\star}$ \\
Serum creatinine $(\mathrm{mg} / \mathrm{dl})$ & $1.78 \pm 0.91$ & $2.54 \pm 1.66$ & $0.834^{*}$ \\
Hb $(\mathrm{gm} / \mathrm{dl})$ & $10.97 \pm 1.60$ & $11.13 \pm 2.50$ & $0.064^{*}$ \\
Serum albumin & $26.10 \pm 9.06$ & $33.29 \pm 8.60$ & $0.585^{*}$ \\
\hline
\end{tabular}

NDRD—Non diabetic renal disease, DN-Diabetic nephropathy; ${ }^{\star}$ Unpaired " $t$ " test was done to measure the level of significance.

Table 6. Glycemic status of patients among different type of renal disease.

\begin{tabular}{cccc}
\hline \multirow{2}{*}{ Variables } & \multicolumn{2}{c}{ Groups } & p value \\
\cline { 2 - 3 } & NDRD & DN & 0.693 \\
FBS (mmol/L) & $7.69 \pm 3.07$ & $8.23 \pm 3.68$ & 0.384 \\
2HABF (mmol/L) & $11.20 \pm 3.27$ & $9.95 \pm 2.40$ & 0.630 \\
HbAlc (\%) & $7.35 \pm 1.42$ & $7.65 \pm 1.03$ & \\
\hline
\end{tabular}

NDRD—Non diabetic renal disease, DN-Diabetic nephropathy; Unpaired " $\mathrm{t}$ " test was done to measure the level of significance.

Table 7. Diabetic retinopathy among the study subjects $(\mathrm{n}=38)$.

\begin{tabular}{cccc}
\hline & \multicolumn{2}{c}{ Diabetic retinopathy } & \multirow{2}{*}{ p value } \\
\cline { 2 - 3 } & Present & Absent & $0.025^{*}$ \\
NDRD & $4(50.0 \%)$ & $27(90.0 \%)$ & \\
DN & $4(50.0 \%)$ & $3(10.0 \%)$ & \\
\hline
\end{tabular}

NDRD—Non diabetic renal disease, DN—Diabetic nephropathy. Values in the parenthesis denote corresponding percentage. ${ }^{*}$ We used Fisher's exact test as statistical method. 
Table 8. Hematuria in different types of renal involvement $(n=38)$.

\begin{tabular}{cccc}
\hline \multirow{2}{*}{ Hematuria } & \multicolumn{2}{c}{ Groups } & \multirow{2}{*}{ p value } \\
\cline { 2 - 3 } & NDRD & DN & $0.002^{\star}$ \\
\hline Present & $28(90.3 \%)$ & $2(28.6 \%)$ & \\
Absent & $3(9.7 \%)$ & $5(71.4 \%)$ & \\
\hline
\end{tabular}

NDRD-Non diabetic renal disease, DN-Diabetic nephropathy. Values in the parenthesis denote corresponding percentage; ${ }^{*}$ We used Fisher's exact test as statistical method.

Table 9. Multivariate logistic regression of risk factors for non-diabetic renal disease.

\begin{tabular}{|c|c|c|c|c|}
\hline & \multirow{2}{*}{$\mathrm{p}$ value } & \multirow{2}{*}{ OR } & \multicolumn{2}{|c|}{$95 \% \mathrm{CI}$} \\
\hline & & & Lower & Upper \\
\hline Duration of DM & 0.766 & 0.634 & 0.032 & 12.765 \\
\hline Absence of diabetic retinopathy & 0.026 & 28.557 & 1.503 & 542.486 \\
\hline Presence of hematuria & 0.701 & 0.482 & 0.012 & 20.097 \\
\hline Serum creatinine & 0.176 & 2.246 & 0.695 & 7.253 \\
\hline $24 \mathrm{~h}$ urinary total protein & 0.669 & 0.918 & 0.620 & 1.359 \\
\hline
\end{tabular}

OR—Odds ratio, CI-Confidence interval, DM-Diabetes mellitus.

\section{Discussion}

Diabetic nephropathy (DN) is the leading cause of end stage renal disease and is associated with increased cardiovascular mortality [14]. Diabetic nephropathy is not only the renal disease in patients with type $2 \mathrm{DM}$, a wide spectrum of non diabetic renal disease (NDRD) including both glomerular and tubulointerstitial lesions can occur [15] [16]. To observe the frequency and histological pattern of NDRD in diabetic patients with proteinuria and to explore their association with clinical and laboratory parameters, we had evaluated thirty eight (38) hospital admitted patients in Department of Nephrology, BSMMU, Dhaka, Bangladesh.

In this study total 38 cases of type 2 diabetic patients on the basis of inclusion criteria were selected. On the basis of histological evidence, these 38 patients were divided into two groups: NDRD (group-I) and DN (group-II). Among them thirty one patients (81.6\%) were placed into Group-I (NDRD) and seven patients (18.4\%) into Group-II (Isolated DN) according to histological findings of renal biopsy specimens. Mean $( \pm \mathrm{SD})$ age of the patients were $43.45 \pm 9.99$ years in the NDRD and $41.57 \pm 9.50$ years in the DN group.

It has been observed that more than two third of the patients $(81.6 \%)$ had NDRD. This result was similar to that reported in studies where histologically confirmed NDRD was found in more than $50 \%$ of diabetics who underwent renal biopsy namely India (64\%), USA (72.5\%) and China (75.5\%). But different from other studies where the prevalence of NDRD was around $12.3 \%-33.3 \%$ [9] [17] [18] [19] [20] [21]. This large variation was presumably due to the different selection criteria for doing renal biopsy in these patients.

In current study 21 (55.3\%) patients were male and 17 (44.7\%) patients were 
female. Male female ratio in NDRD group was 1.0:0.93 and in DN group was 1.0:0.40. So male sex was more common in both group. Wilfred et al. found that female was more common in diabetics with NDRD rather than diabetics with isolated DN [22].

We found that membranoproliferative glomerulonephritis 8 (21.1\%) was the most common NDRD in our study, followed by membranous nephropathy 7 (18.4\%), diffuse proliferative glomerulonephritis 6 (15.8\%), mesangial proliferative glomerulonephritis 5 (13.2\%), focal segmental glomerulosclerosis 3 (7.9\%), IgA nephropathy 1 (2.6\%), minimal change disease 1 (2.6\%) cases. These results were quiet similar to previous study as Prakash et al. showed that membranous nephropathy was the most common NDRD in diabetic patients on renal biopsy [16].

Soni et al. in their study observed that most common NDRD were acute interstitial nephritis (18.1\%), followed by post infectious glomerulonephritis (17.24\%), membranous nephropathy (11.20\%) and focal segmental glomerulosclerosis (7.75\%) [9].

In this study it has been observed that cases in the NDRD group had shorter duration of DM, this being similar to that of previous studies as showed that shorter duration of diabetes, absence of retinopathy, presence of microscopic hematuria, and active urinary sediment were markers associated with NDRD in type 2 diabetes with clinical renal disease [9] [13] [22]-[27].

In this study there was no statistically significant difference had observed for serum creatinine, serum albumin, hemoglobin levels and proteinuria between the two groups which were consistent with other studies [10] [11] [22].

Regarding glycemic status of the study subjects: mean $( \pm \mathrm{SD})$ fasting blood sugar (FBS-mmol/L), 2-hour after postprandial blood sugar (2HABF-mmol/L) and glycosylated hemoglobin (HbAlc -\%) among NDRD patients was $7.69 \pm$ $3.07 \mathrm{mmol} / \mathrm{L}, 11.20 \pm 3.27 \mathrm{mmol} / \mathrm{L}$ and $7.35 \% \pm 1.42 \%$ which was $8.23 \pm 3.68$ $\mathrm{mmol} / \mathrm{L}, 9.95 \pm 2.40 \mathrm{mmol} / \mathrm{L}$ and $7.65 \% \pm 1.03 \%$ among $\mathrm{DN}$ patients respectively. It has been observed that there was no significant difference in glycemic status between NDRD and DN groups. This finding was also consistent with previous study [23].

The current study showed that diabetic retinopathy was absent in 30 cases, of whom 27 (90\%) cases had NDRD. Diabetic retinopathy was seen more frequent among patients with DN than with NDRD. Therefore the absence of retinopathy was a significant predictor of NDRD in this study and that was similar to other studies [15] [22].

Hematuria was present in 30 patients in this study; among them 28 cases had NDRD and 2 cases had DN. Hence the presence of hematuria in a patient with diabetes could be a good predictor of NDRD. Wilfred et al. also stated that hematuria was a significant predictor for NDRD [22].

It has been observed that among diabetic patients with proteinuria, the absence of diabetic retinopathy was the most significant predictor for NDRD and had the highest odds ratio. This observation was consistent with previous studies 
as reported that shorter duration of diabetes and absence of retinopathy were independent predictors of NDRD in diabetic patients with overt proteinuria [24] [25] [26].

\section{Conclusion}

The frequency of non-diabetic renal disease (NDRD) in type 2 diabetic patients other than diabetic nephropathy was relatively high. It has been observed that membranoproliferative glomerulonephritis and membranous nephropathy were more common in NDRD. Absence of diabetic retinopathy, presence of hematuria and shorter duration of DM were markers associated with NDRD in type 2 diabetes mellitus, which were important clinical indications for renal biopsy in diabetic patients with proteinuria.

\section{Limitations of Study}

It was a single centre study with relatively small sample size.

\section{Recommendations}

To identify the correct incidence and histological pattern of non diabetic renal disease (NDRD) in diabetic patients with proteinuria a large scale, multi-center study will be needed.

\section{Conflicts of Interest}

The authors declare no conflicts of interest regarding the publication of this paper.

\section{References}

[1] Zhou, J., Chen, X., Xie, Y., Li, J., Yamanaka, N. and Tong, X. (2007) A Differential Diagnostic Model of Diabetic Nephropathy and Non-Diabetic Renal Diseases. Nephrology Dialysis Transplantation, 23, 1940-1945. https://doi.org/10.1093/ndt/gfm897

[2] National Institutes of Health (2010) US Renal Data Systems: USRDS 2010 Annual Data Report: Atlas of End Stage Renal Disease in the United States. http://www.usrds.org/2010/view/default.asp

[3] Gross, J.L., de Azevedo, M.J., Silveiro, S.P. and Canani, L.H. (2005) Diabetic Nephropathy: Diagnosis, Prevention, and Treatment. Diabetes Care, 28, 64-76. https://doi.org/10.2337/diacare.28.1.164

[4] Mogensen, C.E. and Christensen, C.K. (1984) Predicting Diabetic Nephropathy in Insulin-Dependent Patients. New England Journal of Medicine, 311, 89-93. https://doi.org/10.1056/NEJM198407123110204

[5] Andersen, A.R., Christiansen, J.S., Andersen, J.K., Kreiner, S. and Deckert, T. (1983) Diabetic Nephropathy in Type 1 (Insulin-Dependent) Diabetes: An Epidemiological Study. Diabetologia, 25, 496-501. https://doi.org/10.1007/BF00284458

[6] He, F., Xia, X., Wu, X.F., Yu, X.Q. and Huang, F.X. (2013) Diabetic Retinopathy in Predicting Diabetic Nephropathy in Patients with Type 2 Diabetes and Renal Disease: A Meta-Analysis. Diabetologia, 3, 457-466. 
https://doi.org/10.1007/s00125-012-2796-6

[7] Prakash, J., Lodha, M., Singh, S.K., Vohra, R. and Raja, R. (2007) Diabetic Retinopathy Is a Poor Predictor of Type of Nephropathy in Proteinuric Type 2 Diabetic Patients. The Journal of the Association of Physicians of India, 55, 412-416.

[8] Korbet, S.M. (2002) Percutaneous Renal Biopsy. Seminars in Nephrology, 22, 254-267. https://doi.org/10.1053/snep.2002.31713

[9] Soni, S.S., Gowrishankar, S., Kishan, A.G. and Raman, A. (2006) Non Diabetic Renal Disease in Type 2 Diabetes Mellitus. Nephrology, 11, 533-537.

https://doi.org/10.1111/j.1440-1797.2006.00681.X

[10] Mak, S.K., Gwi, E., Chan, K.W., Wong, P.N., Lo, K.Y., Lee, K.F. and Wong, A.K. (1997) Clinical Predictors of Non-Diabetic Renal Disease in Patients with Non-Insulin Dependent Diabetes Mellitus. Nephrology, Dialysis, Transplantation, 12, 2588-2591. https://doi.org/10.1093/ndt/12.12.2588

[11] Ghani, A.A., Al Waheeb, S., Al Sahow, A. and Hussain, N. (2009) Renal Biopsy in Patients with Type 2 Diabetes Mellitus: Indications and Nature of the Lesions. Annals of Saudi Medicine, 29, 450-453. https://doi.org/10.4103/0256-4947.57167

[12] Prakash, J. (2013) Non-Diabetic Renal Disease (NDRD) in Patients with Type 2 Diabetes Mellitus (Type 2 DM). Journal of the Association of Physicians of India, 61, 194-199.

[13] Chang, T.I., Park, J.T., Kim, J.K., Kim, S.J., Oh, H.J., Yoo, D.E., Han, S.H., Yoo, T.H. and Kang, S.W. (2011) Renal Outcomes in Patients with Type 2 Diabetes with or without Coexisting Non-Diabetic Renal Disease. Diabetes Research and Clinical Practice, 92, 198-204. https://doi.org/10.1016/j.diabres.2011.01.017

[14] John, G.T., Date, A., Korula, A., Jeyaseelan, L., Shastry, J.C. and Jacob, C.K. (1994) Nondiabetic Renal Disease in Noninsulin-Dependent Diabetics in a South Indian Hospital. Nephron, 67, 441-443. https://doi.org/10.1159/000188019

[15] Valmadrid, C.T., Klein, R., Moss, S.E. and Klein, B.E. (2000) The Risk of Cardiovascular Disease Mortality Associated with Microalbuminuria and Gross Proteinuria in Persons with Older-Onset Diabetes Mellitus. Archives of Internal Medicine, 160, 1093-1100. https://doi.org/10.1001/archinte.160.8.1093

[16] Prakash, J., Gupta, T., Prakash, S. and Bhushan, P. (2015) Non-Diabetic Renal Disease in Type 2 Diabetes Mellitus: Study of Renal-Retinal Relationship. Indian Journal of Nephrology, 25, 222. https://doi.org/10.4103/0971-4065.144420

[17] Das, U., Dakshinamurty, K.V., Prayaga, A. and Uppin, M.S. (2012) Nondiabetic Kidney Disease in Type 2 Diabetic Patients: A Single Center Experience. Indian Journal of Nephrology, 22, 358. https://doi.org/10.4103/0971-4065.103912

[18] Pham, T.T., Sim, J.J., Kujubu, D.A., Liu, I.L. and Kumar, V.A. (2007) Prevalence of Nondiabetic Renal Disease in Diabetic Patients. American Journal of Nephrology, 27, 322-328. https://doi.org/10.1159/000102598

[19] Li, H., Li, X.W., Huang, Q.Y., Ye, W.L., Duan, L. and Li, Y. (2003) Non-Diabetic Renal Disease in Type II Diabetes Mellitus. Acta Academiae Medicinae Sinicae, 25, 101-104.

[20] Beck, M.J., Evans, B.J., Quarry-Horn, J.L. and Kerrigan, J.R. (2002) Type 2 Diabetes Mellitus: Issues for the Medical Care of Pediatric and Adult Patients (Featured CME Topic: Pediatrics). Southern Medical Journal, 95, 992-1001. https://doi.org/10.1097/00007611-200295090-00007

[21] Chawarnkul, O., Vareesangthip, K., Ongajyooth, L., Cheunsuchon, B. and Parichatikanond, P. (2009) Non-Diabetic Glomerular Disease in Type II DM: 10 Years Experience. Journal of the Medical Association of Thailand, 92, S57-S60. 
[22] Wilfred, D.C., Mysorekar, V.V., Venkataramana, R.S., Eshwarappa, M. and Subramanyan, R. (2013) Nondiabetic Renal Disease in Type 2 Diabetes Mellitus Patients: A Clinicopathological Study. Journal of Laboratory Physicians, 5, 94. https://doi.org/10.4103/0974-2727.119850

[23] Wong, T.Y., Choi, P.C., Szeto, C.C., To, K.F., Tang, N.L., Chan, A.W., Li, P.K. and Lai, F.M. (2002) Renal Outcome in Type 2 Diabetic Patients with or without Coexisting Nondiabetic Nephropathies. Diabetes Care, 25, 900-905.

https://doi.org/10.2337/diacare.25.5.900

[24] Tone, A., Shikata, K., Matsuda, M., Usui, H., Okada, S., Ogawa, D., Wada, J. and Makino, H. (2005) Clinical Features of Non-Diabetic Renal Diseases in Patients with Type 2 Diabetes. Diabetes Research and Clinical Practice, 69, 237-242. https://doi.org/10.1016/j.diabres.2005.02.009

[25] Huang, F., Yang, Q., Chen, L., Tang, S., Liu, W. and Yu, X. (2007) Renal Pathological Change in Patients with Type 2 Diabetes Is Not Always Diabetic Nephropathy: A Report of 52 Cases. Clinical Nephrology, 67, 293-297. https://doi.org/10.5414/CNP67293

[26] Byun, J.M., Lee, C.H., Lee, S.R., Moon, J.Y., Lee, S.H., Lee, T.W., Ihm, C.G. and Jeong, K.H. (2013) Renal Outcomes and Clinical Course of Nondiabetic Renal Diseases in Patients with Type 2 Diabetes. The Korean Journal of Internal Medicine, 28, 565-572. https://doi.org/10.3904/kjim.2013.28.5.565

[27] Yaqub, S., Kashif, W. and Hussain, S.A. (2012) Non-Diabetic Renal Disease in Patients with Type-2 Diabetes Mellitus. Saudi Journal of Kidney Disease and Transplantation, 23, 1000-1007. https://doi.org/10.4103/1319-2442.100882 